\title{
Editorial
}

\section{Ultrasonic imaging of aortic atheroma}

The study of embolism in the cerebral and peripheral circulation requires imaging methods that reveal pathology in some part of the heart or circulation upstream of the occluded vessel. Technological advances over the past 10-15 years, and in particular the development of transoesophageal, epiaortic, and intravascular ultrasound has made it possible to obtain high resolution images of atherosclerotic lesions in arteries, allowing study of coronary, cerebral, and peripheral vessels as well as the aorta. For aortic disease, transoesophageal and epiaortic ultrasonography have the advantage over other imaging methods, such as computerised tomography or magnetic resonance imaging, of providing real-time images that allow for the evaluation of plaque morphology, ulceration, and mobility. The disadvantages are that transoesophageal ultrasound is semi-invasive and has a blind spot (the upper part of the ascending aorta) and epiaortic ultrasound can only be used intraoperatively.

A number of ultrasonically derived classifications of aortic atheroma has been described based on the thickness of aortic plaques and their morphology. In patients with multiple plaques, the thickness of the deepest plaque is measured. Most investigators have graded the aorta as follows: grade I, no disease or minor intimal thickening; grade II, extensive intimal thickening but without discrete measurable plaques; grade III, protruding plaques $<5 \mathrm{~mm}$ thick; grade $\mathrm{IV}$, protruding plaques $\geqslant 5 \mathrm{~mm}$; grade $\mathrm{V}$, any mobile atheroma. ${ }^{12}$ Other variations on the theme are described with diffuse intimal thickness and small protruding plaques generally being considered as "simple" lesions, and large protruding, ulcerated or mobile lesions as "complex".

Partly because it has been easier to study the carotid vessels, the association between carotid disease and cerebral embolism has been extensively documented, and treatment strategies continue to be evaluated. With such a diffuse arterial process, however, one would expect similar embolic associations with disease elsewhere in major arterial vessels, and in particular the aorta. One necropsy series found that fibrous, calcified, ulcerated, and thrombotic atherosclerotic lesions, observed more frequently in the arch and descending aorta than in the ascending aorta, ${ }^{3}$ were associated with a previous embolic event. Pathology in the arch and ascending aorta would be expected to result in cerebral or peripheral embolism, whereas disease in the descending aorta would be expected to lead to peripheral embolism only.

Necropsy and surgical specimens of the aorta and of embolic material reveal atheromatous material, thrombus, and cholesterol crystals. ${ }^{4}$ Some researchers believe that mobile plaques detected with transoesophageal echocardiography are the fibrous caps of ruptured plaques ${ }^{2}$; however, thrombi may give the same appearance. Diffuse intimal thickening must also be distinguished from intramural haematoma ${ }^{6}$ or layered thrombus in aneurysmal segments. Apart from areas of calcification, the true composition of diseased segments cannot be analysed with presently available techniques but the importance of the composition in determining embolic potential is unknown.

Despite the difficulty in determining the nature of the tissue seen with transoesophageal ultrasonography, there is now an extensive literature on the association of severe atheromatous disease with peripheral or cerebral embolic events. These (usually retrospective) case-control studies uniformly reveal that the presence of aortic atheroma, especially complex lesions, is associated with systemic embolism. Multivariate analysis demonstrates a probable causative role independent of established risk factors. ${ }^{7-9}$ The association is much stronger in elderly patients (clinicians who limit transoesophageal echocardiography to selected young patients with cryptogenic stroke will not have noted it) and in those with no other obvious cause of stroke. However, the complexity of aortic atherosclerosis is associated with age, hypertension, diabetes, smoking, hyperlipidaemia, carotid disease, atrial fibrillation, intraaortic spontaneous contrast echoes, and even cardiac sources of embolism. All of these are established risk factors for vascular events, and in the individual elderly patient with a stroke several disorders may be detected, making it difficult to determine the cause. Nonetheless, the fact that an independent role in embolism is found suggests that atherosclerotic debris from the aorta may be responsible for more clinical events than has hitherto been realised.

Some investigators have used the association of aortic atheroma with disease elsewhere in the circulation to predict whether patients have significant coronary disease. ${ }^{10} 11$ Not surprisingly, the more extensive and the more complex the aortic disease the more likely the patient is to have prognostically important coronary disease. It is unlikely that these studies will have any significant bearing on the future non-invasive or invasive assessment of coronary disease, but they may be useful in a negative sense in that patients with normal aortas are very unlikely to have significant coronary atheroma. It is proposed that such information may preclude the requirement for coronary angiography in the assessment of patients with valve or aortic disease. An important association has also been noted between aortic disease and embolic events at the time of insertion of cardiac catheters and intra-aortic balloon pumps from the femoral artery; it is suggested that if coronary angiography is required in these patients it should be performed via the brachial route. ${ }^{12}$ Further study is required to determine which groups of patients should be considered for transoesophageal echocardiography before invasive tests or balloon pumping.

Although many factors have been implicated in the development of neurological damage after cardiac surgery, atheromatous embolisation may be a particular problem faced by patients having coronary artery bypass surgery. Transoesophageal and transcranial Doppler ultrasonography studies suggest that embolisation occurs most frequently with cannulation of the aorta to institute cardiopulmonary bypass, ${ }^{1}$ and after release of aortic cross-clamps and partial occlusion clamps. ${ }^{13}$ Whether these and epiaortic techniques can identify patients who should be treated by alternative surgical methods to reduce neurological complications remains to be seen.

Although aortic atheromatous embolisation appears to be a very real problem, some would quite rightly argue that ultrasound techniques have allowed research into this area but have not yet revealed any successful strategies to prevent recurrences. Moreover, little is known about the natural history of the different grades of atherosclerosis. 
Tunick et al reported a 33\% vascular event rate over a mean 14 month follow up period in a group of 42 patients with plaques $\geqslant 5 \mathrm{~mm}$ or with mobile components. ${ }^{14}$ They also suggested that there might be two forms of embolisation from such plaques: large thrombotic emboli leading to large vessel occlusion resulting in stroke, limb ischaemia and organ infarction; and diffuse cholesterol embolisation resulting in renal failure and the blue toe syndrome. Montgomery and colleagues studied patients who had repeat transoesophageal studies 6-22 months apart and found that individual lesions changed morphology over time. ${ }^{2}$ Whereas the underlying intimal thickness remained stable, mobile components were often found to have resolved (without overt clinical events), whereas other plaques had developed new mobile components. The authors suggested that a strategy of plaque prevention or stabilisation with antithrombotic or lipid lowering treatment might confer the greatest benefit in terms of reduction of clinical events. The French Study Group report of 331 patients 60 years or older $(64 \%$ of whom were treated with antiplatelet agents, $18 \%$ with anticoagulants) revealed an association between the rate of future cerebral events and the depth of plaque - those with plaques $\geqslant 4 \mathrm{~mm}$ had a recurrence rate of 12 for every 100 patient-years despite the selected treatment. ${ }^{15}$

A number of treatment strategies can be considered for patients presenting with otherwise unexplained embolic events and protruding or complex aortic atheromatous disease. Although aspirin and warfarin have not prevented the development of new complex lesions with mobile components or further clinical events, ${ }^{2} 1415$ these observations do not preclude some degree of protective effect. On the other hand, there may be some distinct risks with anticoagulant drugs. Although they may prevent the formation or allow safe resolution of thrombi, there has been a worry that they might promote bleeding into plaques with further ulceration, or that they might remove the thrombin coating from ulcerated plaques and facilitate cholesterol embolisation. ${ }^{16}$ Moreover, whereas apparently successful resolution of mobile components has been reported in some patients, anticoagulant treatment has been ineffective in others. Anecdotal reports of apparently successful thrombolysis in this situation ${ }^{17}$ must be balanced by a worry that this also may enhance embolisation. ${ }^{18}$ Successful surgical approaches with aortic replacement or debridement have also been used in patients with multiple cerebral events or widespread peripheral embolisation, ${ }^{19} 20$ but whether benefits outweigh risks is unknown.

In conclusion, the development of ultrasonic imaging methods has revealed that atherosclerotic debris within the aorta is probably responsible for more embolic events than has hitherto been accepted. The association with embolism is greatest for older patients and for those with more complex disease such as large protruding plaques or plaques with ulceration or mobile components. The natural history of these plaques appears to be dynamic, but may lead to embolisation of either thrombus or cholesterol laden debris. Large prospective studies of different treatment strategies are needed to determine whether plaque stabilisation can be achieved and the risk of recurrent embolisation reduced. Until such information is available, the widespread use of transoesophageal echocardiography in elderly patients with stroke cannot be advocated. If it is performed the physician will be in the position of occasionally finding alarming disease and not knowing what to do about it.

Consultant Cardiologist,

M A DE BELDER

South Cleveland Hospital,

Marton Road,

Middlesbrough TS4 3BW, UK

1 Katz ES, Tunick PA, Rusinek H, et al. Protruding aortic atheromas predict stroke in elderly patients undergoing cardiopulmonary bypass: a review of our experience with intraoperative transesophageal echocardiography. $7 \mathrm{Am}$ our experience with intraoper

2 Montgomery DH, Ververis JJ, McGorisk G, et al. Natural history of severe atheromatous disease of the thoracic aorta: a transoesophageal study. $\mathcal{f} \mathrm{Am}$ Coll Cardiol 1996;27:95-101.

3 Khatibzadeh M, Mitusch R, Stierle U, et al. Aortic atherosclerotic plaques as a source of embolism. $\mathcal{F}$ Am Coll Cardiol 1996;27:664-9.

4 Amarenco P, Cohen A, Baudrimont M, et al. Transesophageal echocardiographic detection of aortic arch disease in patients with cerebral infarction. Stroke 1992;23:1005-9.

5 Aldrich HR, Girardi LN, Bush HL, et al. Recurrent systemic embolization caused by aortic thrombi. Ann Thorac Surg 1994;57:466-8.

6 Nienaber CA, von Kodolitsch Y, Petersen B, et al. Intramural hemorrhage of the thoracic aorta: diagnostic and therapeutic implications. Circulation 1995;92:1465-72.

7 Amarenco P, Cohen A, Tzourio C, et al. Atherosclerotic disease of the aortic arch and the risk of ischemic stroke. N Engl f Med 1994;331:1474-9.

8 Davila-Roman VG, Barzilai B, Wareing TH, et al. Atherosclerosis of the ascending aorta. Prevalence and role as an independent predictor of cerebrovascular events in cardiac patients. Stroke 1994;25:2010-16.

9 Jones EF, Kalman JM, Calafiore P, et al. Proximal aorta atheroma. An independent risk factor for cerebral ischemia. Stroke 1995;26:218-24.

10 Tribouilloy C, Shen WF, Peltier M, et al. Noninvasive prediction of coronary artery disease by transesophageal echocardiographic detection of thoracic aortic plaque in valvular heart disease. Am $\mathcal{F}$ Cardiol 1994;74:258-60.

11 Parthenakis F, Skalidis E, Simantirakis E, et al. Absence of atherosclerotic lesions in the thoracic aorta indicates absence of significant disease. Am $\mathcal{F}$ Cardiol 1996;77:1118-21.

12 Karalis DG, Quinn V, Victor MF, et al. Risk of catheter-related emboli in patients with atherosclerotic debris in the thoracic aorta. Am Heart $\mathcal{F} 1996$; 131:1149-55.

13 Barbut D, Yao FS, Hager DN, et al. Comparison of transcranial Doppler ultrasonography and transesophageal echocardiography to monitor emboli ultrasonography and transesophageal echocardiography to mo
during coronary artery bypass surgery. Stroke 1996;27:87-90.

14 Tunick PA, Rosenzweig BP, Katz ES, et al. High risk for vascular events in patients with protruding aortic atheromas: a prospective study. $\mathcal{F} \mathrm{Am}$ Coll Cardiol 1994;23:1085-90

15 The French Study of Aortic Plaques in Stroke Group. Atherosclerotic disease of the aortic arch as a risk factor for recurrent ischemic stroke. $N$ Engl F Med 1996;334:1216-21.

16 Hilton TC, Menke D, Blackshear JL. Variable effect of anticoagulation in the treatment of severe protruding atherosclerotic aortic debris. Am Heart $\mathcal{f}$ 1994;127:1645-7.

17 Hausmann D, Gulba D, Bargheer K, et al. Successful thrombolysis of an aortic arch thrombus in a patient after mesenteric embolism. $N$ Engl f Med 1992;327:500-1.

18 Karalis DG, Kaulback KW, Ross JJ Jr, et al. Spontaneous arterial embolization after thrombolytic therapy for acute myocardial infarction: the role of tion after thrombolytic therapy for acute myocardial infarction: the role of transesor

19 Swanson SJ, Cohn LH. Excision of focal aortic arch atheroma using deep hypothermic circulatory arrest. Ann Thorac Surg 1995;60:457-8.

20 Bojar RM, Payne DD, Murphy RE, et al. Surgical treatment of systemic atheroembolism from the thoracic aorta. Ann Thorac Surg 1996;61:138993. 